\title{
Bone Loss in Women with Type 1 Diabetes
}

\author{
Maj-Britt Tanderup Jørgensen ${ }^{1}$, Jesper Olund Christensen ${ }^{2,3}$, Ole Lander Svendsen ${ }^{1,2,3 *}$ \\ ${ }^{1}$ Department of Endocrinology I, Bispebjerg University Hospital, Copenhagen, Denmark \\ ${ }^{2}$ Steno Diabetes Center, Gentofte, Denmark \\ ${ }^{3}$ Center for Clinical and Basic Research, Ballerup, Denmark \\ Email: *ole.lander.svendsen@regionh.dk
}

Received 5 September 2015; accepted 25 October 2015; published 28 October 2015

Copyright (C) 2015 by authors and Scientific Research Publishing Inc.

This work is licensed under the Creative Commons Attribution International License (CC BY). http://creativecommons.org/licenses/by/4.0/

(c) (7) Open Access

\begin{abstract}
Background: Although osteoporosis has been investigated and debated in the diabetic population over the past decades, very little is known about the spontaneous changes in bone mineral density (BMD) and biochemical markers of bone turnover in pre- and postmenopausal type 1 diabetic (T1DM) women over time. Aim: To measure spontaneous changes in BMD and biochemical markers of bone turnover in pre- and postmenopausal T1DM women. Subjects: 53 T1DM women (31 premenopausal and 22 postmenopausal) from the outpatient clinic were enrolled in the study in 1993 and 35 (22 premenopausal, 13 postmenopausal) were reexamined in 1997. Method: BMD was measured at femoral neck (f.n.), spine $\left(L_{2}-L_{4}\right)$, total body and forearm with DXA or SXA in 53 T1DM women. 4 years later a re-scan was carried out on 35 T1DM. Results: In premenopausal subjects a yearly decrease less than $1 \%$ at f.n., spine, forearm and total body was observed, though only statistically significant (s.s.) at f.n., $p \leq 0.05$. In postmenopausal subjects a s.s. decrease less than $2 \%$ was observed at f.n., forearm and total body, $p \leq 0.05$. In general, osteopenic or osteoporotic values were observed at the measured skeletal sites. Only at f.n. a lower s.s. BMD compared to age-matched reference women was seen. Conclusion: Small or non-significant changes in BMD and biochemical markers of bone turnover were observed in pre- and postmenopausal T1DM subjects after a 4-year period.
\end{abstract}

\section{Keywords}

Bone Turnover, Bone Mineral Density, Biochemical Markers of Bone Turnover, Premenopausal, Postmenopausal

\section{Introduction}

The interest in osteoporosis has been steadily increasing over the past decade. It is considered to be a significant

${ }^{*}$ Corresponding author. 
health issue due to high morbidity, mortality and aging populations in developed countries [1]. In the diabetic population, osteoporosis has been investigated and debated since Albright [2] first reported on the topic postmenopausal osteoporosis. Since then, BMD in patients with type 1 diabetes has been reported to be higher [3] [4], similar [5]-[7] or lower [8] compared to control groups, though the majority of the studies report a slight reduction in BMD at one or more skeletal sites where osteoporotic fractures most frequently occur [9]-[11]. The majority of studies focus on premenopausal diabetic women, whilst only a small number of studies focus on postmenopausal diabetic women. The design of these studies is often based upon a cross-sectional approach rather than a longitudinal approach. Very little is therefore known about BMD in postmenopausal type 1 diabetic women or spontaneous changes in BMD and biochemical markers of bone turnover in pre- and postmenopausal type 1 diabetic women.

The aim of this study was to investigate the spontaneous changes in BMD and biochemical markers of bone turnover after a 4-year period, as well as the occurrence of osteopenia/osteoporosis in a group of type 1 and type 2 diabetic women. This was done by a follow-up study in 1997 on a group of pre- and postmenopausal diabetic women, previously studied in 1993 [11].

\section{Method}

\subsection{Subjects}

Female patients with diabetes mellitus were recruited from the outpatient clinic at the Steno Diabetes Center by reviewing patient files. Exclusion criteria were; random glucose $>20 \mathrm{mmol} / \mathrm{l}$, ketonuria, pregnancy, age $<18$ years, hysterectomia, nephropathy (albuminuria $>300 \mathrm{mg} / \mathrm{day}$, serum creatinine $>0.15 \mathrm{mmol} / \mathrm{l}$ ), having a major disease other than diabetes mellitus, surgery or current medication (other than anti-diabetic medication) with known effects on bone metabolism. Of 215 potentially eligible patients contacted by questionnaire and invited to participate, 15 could not be reached, 104 did not wish to participate and 11 were excluded based upon the exclusion criteria. Thus 85 female patients were enrolled in the study in 1993.

The subjects were divided based upon type of diabetes and menopausal status (patients for whom more than 6 months had passed since their last menstruation without other known causes were classified as postmenopausal). Of the 85 subjects 53 had type 1 diabetes ( 31 were premenopausal and 22 were postmenopausal) and 32 had type 2 diabetes ( 11 were premenopausal and 21 were postmenopausal). In 1997, the 85 subjects were contacted again, 34 could not be reached or did not wish to participate, and thus 51 subjects attended the follow-up visit. Since 1993, 8 had started hormone replacement therapy and were excluded. In this way 35 type 1 diabetic patients (22 premenopausal and 13 postmenopausal) and 8 type 2 diabetic patients ( 2 premenopausal and 6 postmenopausal) were enrolled in the follow-up study. Data from type 2 diabetic women is omitted due to a low follow-up and an insufficient sample group, thus only data from type 1 diabetic women are presented. Three premenopausal women with type 1 diabetes reached menopause between 1993 and 1997, these subjects remained in the premenopausal group. Body composition and fat distribution as well as BMD and bone remodeling rates of the study population from visit 1 have been reported previously [11] [12].

An interview was used to determine anamnestic variables such as concomitant diseases, presence of diabetic complications, use of antidiabetic therapy and use of other medications. The duration of diabetes was calculated with the date of diagnosis serving as the onset. With the subjects wearing light indoor clothes and no shoes, weight was measured to the nearest $0.1 \mathrm{~kg}$ and height to the nearest $0.5 \mathrm{~cm}$. $\mathrm{HbA}_{1 \mathrm{c}}$ values were obtained as the last recorded value in the patient file (Table 1). The study was approved by the Copenhagen County Ethical Committee and carried out in accordance with the Helsinki Declaration II.

\subsection{BMD Measurements}

BMD of the lumbar spine $\left(\mathrm{L}_{2}-\mathrm{L}_{4}\right.$, anteroposterior projection), left femoral neck and total body were measured by dual-energy X-ray absorptiometry (DXA) (QDR 2000 X-ray bone densitometer, Hologic Waltham, MA; fan beam software versions 4.66A:1, 4.56A:1 and 5.54A respectively). BMD in the non-dominant forearm was measured proximal to the site where the distance between radius and ulna is $8 \mathrm{~mm}$ by single $\mathrm{X}$-ray absorptiometry (SXA) (DTX-100, Osteometer, Roedovre, Denmark; software version 1.30). BMD measurements were expressed in $\mathrm{g} / \mathrm{cm}^{2}$. For each BMD measurement at the femoral neck, spine and total body, a t-score was calculated according to the formula $t$-score $=\left[\right.$ patient's BMD value $\left(\mathrm{g} / \mathrm{cm}^{2}\right)-$ mean BMD of a reference group of sex- 
Table 1. Demographic and diabetes variables for type 1 diabetic women.

\begin{tabular}{ccc}
\hline & Premenopausal & Postmenopausal \\
\cline { 2 - 3 } Age (years) & $(\mathrm{n}=22)$ & $(\mathrm{n}=13)$ \\
Years since menopause & $40(28-49)$ & $61(52-76)$ \\
Body mass index $\left(\mathrm{kg} / \mathrm{m}^{2}\right)$ & - & $11.5(2.5-22)$ \\
Diabetes duration (years) & $23(20-28)$ & $22(19-24)$ \\
$\mathrm{HbA}_{\mathrm{lc}}(\mathrm{mmol} / \mathrm{l})$ & $14(0.9-28)$ & $25(5-45)$ \\
No diabetic complications & $8.3(6.1-10.5)^{* 1}$ & $9.4(4.4-10.6)^{* 2}$ \\
One diabetic complication & $\mathrm{n}=18(82 \%)$ & $\mathrm{n}=9(69 \%)$ \\
Two diabetic complications & $\mathrm{n}=4(18 \%)$ & $\mathrm{n}=3(23 \%)$ \\
\hline
\end{tabular}

${ }^{* 1}: \mathrm{n}=20,{ }^{* 2}: \mathrm{n}=12$. Values are median and range.

matched 30 year old healthy individuals $\left.\left(\mathrm{g} / \mathrm{cm}^{2}\right)\right]$ : standard deviation (SD) of a reference group $\left(\mathrm{g} / \mathrm{cm}^{2}\right)$ for the postmenopausal subjects. T-scores for the forearm were derived from software provided by the manufacturer for the postmenopausal subjects. Osteopenia was defined as a t-score of 1 to $2.5 \mathrm{SD}$ below the mean of the reference group. Osteoporosis was defined as a t-score of 2.5 or more below the mean of a reference group. Furthermore a $\mathrm{z}$-score was calculated according to the formula $\mathrm{z}$-score $=\left[\right.$ patient's BMD value $\left(\mathrm{g} / \mathrm{cm}^{2}\right)-$ mean BMD of a reference group of age and sex-matched healthy individuals $\left.\left(\mathrm{g} / \mathrm{cm}^{2}\right)\right]$ : SD of a reference group $\left(\mathrm{g} / \mathrm{cm}^{2}\right)$ for each BMD measurements at the femoral neck, spine and total body for the postmenopausal subjects. Z-scores for the forearm were derived from software provided by the manufacturer for the postmenopausal subjects.

\subsection{Biochemical Measurements}

As a marker of bone formation, serum osteocalcin was measured by an enzyme-linked immunosorbent assay (ELISA) according to the manufacturer's instructions. The assay measured the N-terminal-mid fragment as well as the intact human osteocalcin in serum [13]. As a marker of bone resorption, the Crosslaps ELISA (Osteometer A/S, Roedovre, Denmark) was used according to the manufacturer's instructions. The Crosslaps ELISA is based on an immobilized synthetic peptide with an amino acid sequence specific for a part of the C-telopeptide of the $\mathrm{C} \alpha \mathrm{I}$ chain of type I collagen. All determinations were corrected for creatinine excretion [13]. Reference values for biochemical markers of bone absorption and bone resorption were obtained from Ravn et al., who studied BMD and bone remodeling in 979 healthy white women aged 30 - 75 years [13] (Table 2).

\subsection{Statistical Analyses}

A paired t-test (two-tailed) was used to compare BMD at visit 1 and visit $2, p \leq 0.05$ was considered statistically significant. A goodness-of-fit test was carried out to ensure that the distribution was Gaussian. A two-tailed t-test was used to compare the level of biomarkers at visit 1 and 2 . The values were logarithmically transformed since the distributions did not approximate Gaussian. An unpaired t-test (assuming unequal variance and unequal observations (Aspin-Welch test)) was used to compare the level of biomarkers to reference values.

\section{Results}

Demographic characteristics and diabetes variables of pre- and postmenopausal patients at visit 1 appears in Table 1. Table 2 indicates the BMD measurements at different sites. For all sites it appears that BMD measurements were higher in the premenopausal group than in the postmenopausal group. The calculated yearly change in BMD is given in Figure 1(a) and Figure 1(b) for pre- and postmenopausal patients respectively. In the group of premenopausal patients, a yearly decrease of less than $1 \%$ at the femoral neck, spine, forearm and total body was observed, though only statistically significant at the femoral neck. In the group of postmenopausal patients a statistically significant decrease of less than $2 \%$ was observed at the femoral neck, forearm and total body. No statistically significant decrease was observed at the spine. 


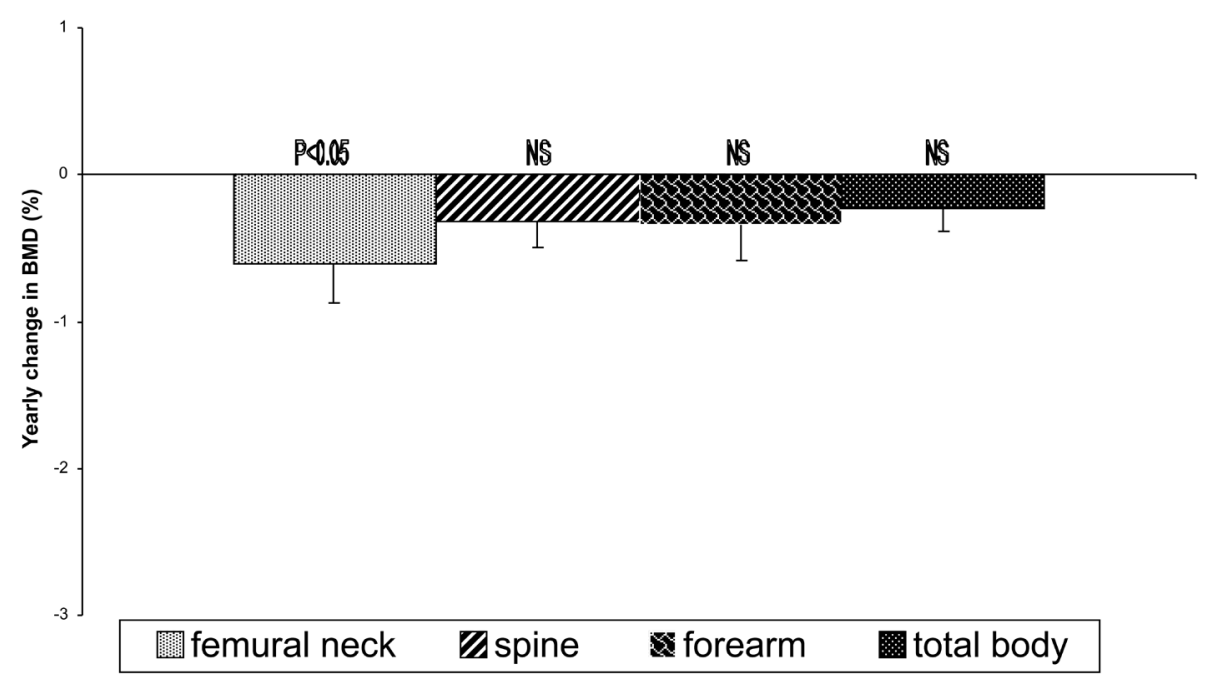

(a)

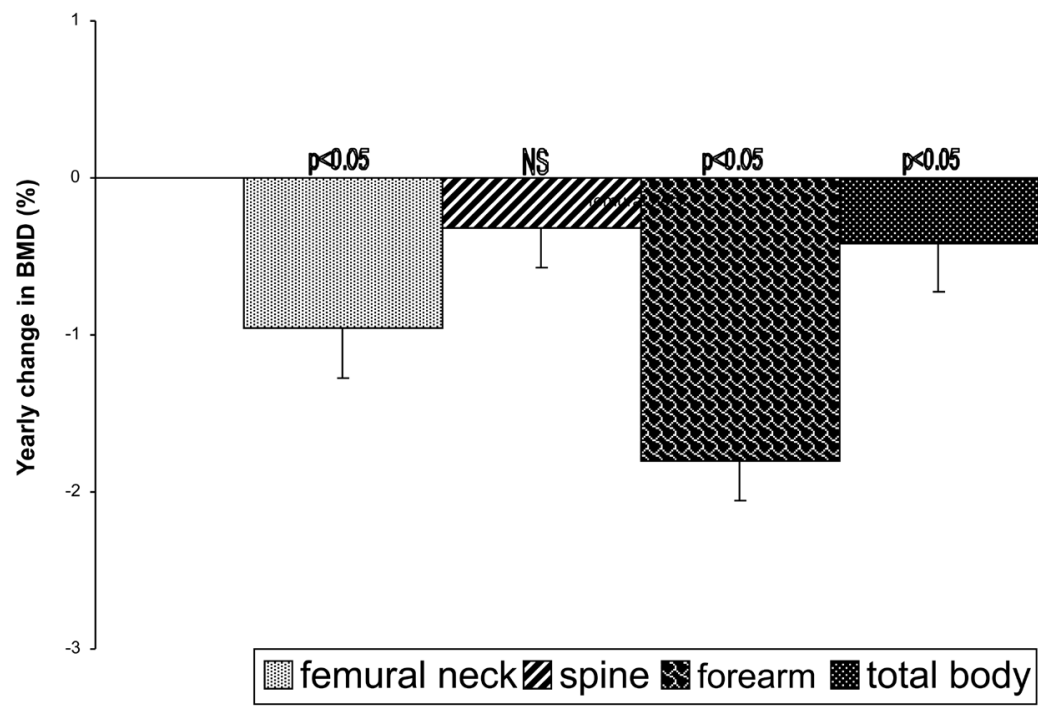

(b)

Figure 1. (a) Changes in BMD in premenopausal type 1 diabetic women. Values are calculated yearly changes in BMD \pm SEM. S: $\leq 0.05$, NS: $p>0.05$; (b) Changes in BMD in postmenopausal type 1 diabetic women. Values are calculated yearly changes in $\mathrm{BMD} \pm \mathrm{SEM}$. S: $\leq 0.05, \mathrm{NS}: p>0.05$.

Table 2. BMD and biochemical markers of bone turnover for type 1 diabetic women.

\begin{tabular}{|c|c|c|c|c|}
\hline & \multicolumn{2}{|c|}{ Premenopausal } & \multicolumn{2}{|c|}{ Postmenopausal } \\
\hline & \multicolumn{2}{|c|}{$\mathrm{n}=22$} & \multicolumn{2}{|c|}{$\mathrm{n}=13$} \\
\hline & 1993 & 1997 & 1993 & 1997 \\
\hline Femural neck $\left(\mathrm{g} / \mathrm{cm}^{2}\right)$ & $0.81 \pm 0.03$ & $0.79 \pm 0.03$ & $0.62 \pm 0.03$ & $0.60 \pm 0.03$ \\
\hline Lumbar spine $\mathrm{L}_{2}-\mathrm{L}_{4}\left(\mathrm{~g} / \mathrm{cm}^{2}\right)$ & $1.09 \pm 0.03$ & $1.08 \pm 0.03$ & $0.95 \pm 0.04$ & $0.94 \pm 0.04$ \\
\hline Forearm (distal) $\left(\mathrm{g} / \mathrm{cm}^{2}\right)$ & $0.47 \pm 0.01^{\wedge}$ & $0.46 \pm 0.01^{\wedge}$ & $0.39 \pm 0.02^{\#}$ & $0.36 \pm 0.02^{\#}$ \\
\hline Total body $\left(\mathrm{g} / \mathrm{cm}^{2}\right)$ & $1.11 \pm 0.02$ & $1.10 \pm 0.02$ & $0.96 \pm 0.03$ & $0.95 \pm 0.26$ \\
\hline Osteocalcin (ng/mL) & $20.4 \pm 1.1^{¥}$ & $18.7 \pm 1.3^{\neq \mp}$ & $31.3 \pm 1.8^{a}$ & $27.8 \pm 2.0^{¥ \S}$ \\
\hline Crosslaps $(\mu \mathrm{g} / \mathrm{mmol})$ & $176.6 \pm 23.9^{* ¥}$ & $206.5 \pm 35.1^{* ¥ \pi}$ & $417.9 \pm 85.1^{\# \infty}$ & $387.1 \pm 74.6^{\# \# \S}$ \\
\hline
\end{tabular}

BMD values and biochemical markers: mean \pm SEM. Reference values for biochemical markers: osteocalcin; pre: $23.7 \pm 0.34 \mathrm{ng} / \mathrm{mL}$, post: $32.1 \pm$ $0.38 \mathrm{ng} / \mathrm{mL}$, crosslaps; pre: $314 \pm 8.3 \mu \mathrm{g} / \mathrm{mmol}$, post: $498 \pm 12.3 \mu \mathrm{g} / \mathrm{mmol} .^{*}: n=20,{ }^{\wedge}: \mathrm{n}=21,{ }^{\#}: \mathrm{n}=12$. Difference from reference values: ${ }^{*}: p \leq 0.05$, ${ }^{\square}: p>0.05$. Difference between visit 1 and visit $2:{ }^{\S}: p \leq 0.05,{ }^{\natural}: p>0.05$. 
In premenopausal diabetic subjects, both bone resorption and bone formation markers were statistically significantly lower than reference values for premenopausal healthy subjects and no statistically significant change between the two visits was observed, see Table 2. In postmenopausal diabetic subjects, resorption and formation markers were not statistically significantly different from the reference values for postmenopausal healthy subjects at visit 1, whereas both resorption and formation markers were statistically significantly lower at visit 2 and moreover a statistically significant decrease between visit 1 and visit 2 was observed, see Table 2.

Postmenopausal patients had in general osteopenic or osteoporotic values at the measured sites. Of the 12 subjects (one subject did not attend visit 2 for the SXA scan) 4 (33\%) subjects had osteopenia at one or more sites (femoral neck, spine or forearm), 8 (67\%) subjects had osteoporosis at one or more sites (femoral neck, spine or forearm) (4 of these had additional osteopenia at one or two sites) and no one had normal BMD at all three sites in 1997. At the femoral neck a statistically significantly lower BMD compared to age-matched reference women was observed (femoral neck: mean z-score $=-1.0(95 \% \mathrm{CI}:-1.4$ to -0.5$)$, spine: mean $\mathrm{z}$-score $=$ $-0.6(95 \% \mathrm{CI}:-0.3$ to +1.2$)$ and forearm: mean $\mathrm{z}$-score $=-0.04(95 \% \mathrm{CI}:-0.8$ to +0.7$)), 7$ out of $12(58 \%)$ subjects had a z-score of $1 \mathrm{SD}$ below the mean of the reference group and $1(8 \%)$ subject had a z-score of $2.5 \mathrm{SD}$ below the mean of the reference group. In the postmenopausal group the change in BMD during the study period had only a minor effect on the initial diagnosis given in 1993. 1 subject changed from normal to osteopenic values at the femoral neck and forearm and 2 subjects changed from osteopenic to osteoporotic values either at the femoral neck or at the forearm. The other 9 subjects did not change diagnostic category.

\section{Discussion}

An annual decrease in BMD lower than $2 \%$ at the femoral neck, forearm and total body was observed in postmenopausal subjects. Thus the study indicates that during the 4 year period no or only small changes in BMD toke place. This is also supported by the fact that biochemical markers of bone resorption and formation were found no different or even lower than for reference women. The comparison with biochemical reference values should be interpreted with caution though as reference values where matched only according to sex and menopausal status, and not according to age. However, it should be noted that these findings are consistent with what others have reported [5] [8]. In premenopausal subjects small or non-significant changes in BMD and biochemical markers of bone turnover were observed. Similar observations was done by Miazgowski et al., who studied changes in BMD and biochemical markers of bone turnover after a 2 year period in 54 male and female type 1 diabetic patients with longstanding diabetes $((\operatorname{mean} \pm \mathrm{SD})$ female; age: $36.9 \pm 8$, BMI: $23.4 \pm 2.5$, duration of diabetes: $16.9 \pm 8$, male; age: $40.5 \pm 9$, BMI: $24.5 \pm 2.3$, duration of diabetes: $16.5 \pm 8$ ) [10]. It is also consistent with the result of another longitudinal study of changes in BMD after a 2.5 year period in 15 type 1 diabetic patients with longstanding diabetes [14]. The sample group of that study was very heterogenic though and comprised male and female, black and white as well as pre- and postmenopausal subjects. In the postmenopausal group, 8 out of 12 subjects had osteoporosis at one or more sites (femoral neck, spine or forearm). Compared to age-matched reference women, BMD was only statistically significantly lower at the femoral neck. It should be noted though that the diabetic subjects were compared to reference women of the same age and ethnicity but not of the same height and weight when z-score was calculated. The z-score should therefore be interpreted with caution. It is known that a $1 \mathrm{SD}$ decrease in BMD measured by DXA is associated with an approximately 2-fold increase in the relative risk of fracture [15]. Something suggests, however, that the risk of hip fracture in postmenopausal type 1 diabetic women is even higher. Nicodemus et al. studied a group of postmenopausal type 1 diabetic women $(n=47)$ aged 61.5 years with long-standing diabetes which showed a 5-33 increased risk of hip fracture [16]. Thus, other risk factors are evident in this population. It appears that changes in BMD solely in postmenopausal type 1 diabetic women have not previously been studied over time. In general the number of studies carried out only with postmenopausal type 1 diabetic subjects is limited and no one has studied the spontaneous changes in BMD and biochemical markers of bone turnover over time. More studies on the topic are therefore desirable.

\section{Conclusion}

Over a 4-year period, no or only small changes in BMD and biochemical markers of bone turnover were observed in pre- and postmenopausal type 1 women. This suggests that bone loss is about the same size as normally seen in non-diabetic healthy women. 


\section{References}

[1] Kanis, J.A. and the WHO Study Group (1994) Assessment of Fracture Risk and Its Application to Screening for Postmenopausal Osteoporosis: Synopsis of a WHO Report. Osteoporosis International, 4, 368-381. http://dx.doi.org/10.1007/BF01622200

[2] Albright, F.A. (1941) Postmenopausal Osteoporosis. JAMA, 116, 2465-2474. http://dx.doi.org/10.1001/jama.1941.02820220007002

[3] Gallacher, S.J., Fenner, J.A.K., Fisher, B.M., et al. (1993) An Evaluation and Bone Density and Turnover in Premenopausale Women with Type 1 Diabetes Mellitus. Diabetic Medicine, 10, 129-133. http://dx.doi.org/10.1111/j.1464-5491.1993.tb00029.x

[4] Lunt, H., Florkowski, C.M., Cundy, T., et al. (1998) A Population-Based Study of Bone Mineral Density in Women with Longstanding Type 1 (Insulin-Dependent) Diabetes. Diabetes Research and Clinical Practice, 40, 31-38. http://dx.doi.org/10.1016/S0168-8227(98)00012-6

[5] Giacca, A., Fassina, A., Caviezel, F., et al. (1988) Bone Mineral Density in Diabetes Mellitus. Bone, 9, 29-36. http://dx.doi.org/10.1016/8756-3282(88)90024-5

[6] Ingberg, C.M., Palmer, M., Åman, J., et al. (2004) Body Composition and Bone Mineral Density in Long-Standing Type 1 Diabetes. Journal of Internal Medicine, 255, 392-398. http://dx.doi.org/10.1046/j.1365-2796.2003.01283.x

[7] Rakic, V., Davis, W.A., Chubb, S.A.P., et al. (2006) Bone Mineral Density and Its Determinants in Diabetes: The Fremantle Diabetes Study. Diabetologia, 49, 863-871. http://dx.doi.org/10.1007/s00125-006-0154-2

[8] Rachon, D., Mysliwska, J., Suchecka-Rachon, K., et al. (2003) Serum Interleukin-6 Levels and Bone Mineral Density at the Femoral Neck in Post-Menopausal Women with Type 1 Diabetes. Diabetic Medicine, 20, 475-480. http://dx.doi.org/10.1046/j.1464-5491.2003.00953.x

[9] Compston, J.E., Smith, E.M., Matthews, C., et al. (1994) Whole Body Composition and Regional Bone Mass in Women with Insulin-Dependent Diabetes Mellitus. Clinical Endocrinology (Oxford), 41, 289-293. http://dx.doi.org/10.1111/j.1365-2265.1994.tb02547.x

[10] Miazgowski, T. and Czekalski, S. (1998) A 2-Year Follow-Up Study on Bone Mineral Density and Markers of Bone Turnover in Patients with Long-Standing Insulin-Dependent Diabetes Mellitus. Osteoporosis International, 8, $399-403$. http://dx.doi.org/10.1007/s001980050082

[11] Christensen, J.O. and Svendsen, O.L. (1999) Bone Mineral in Pre- and Postmenopausal Women with Insulin-Dependent and Non-Insulin-Dependent Diabetes Mellitus. Osteoporosis International, 10, 307-311. http://dx.doi.org/10.1007/s001980050232

[12] Svendsen, O.L. and Hassager, C. (1998) Body Composition and Fat Distribution Measured by Dual-Energy X-Ray Absorptiometry in Premenopausal and Postmenopausal Insulin-Dependent and Non-Insulin-Dependent Diabetes Mellitus Patients. Metabolism, 47, 212-216. http://dx.doi.org/10.1016/S0026-0495(98)90223-2

[13] Ravn, P., Fledelius, C., Rosenquist, C., et al. (1996) High Bone Turnover Is Associated with Low Bone Mass in both Pre- and Postmenopausal Women. Bone, 19, 291-298. http://dx.doi.org/10.1016/8756-3282(96)00201-3

[14] Krakauer, J.C., Mckenna, M.J., Buderer, N.F., et al. (1995) Bone Loss and Bone Turnover in Diabetes. Diabetes, 44, 775-781. http://dx.doi.org/10.2337/diab.44.7.775

[15] Delmas, P.D., Eastell, R., Gernero, P., et al. (2000) The Use of Biochemical Markers of Bone Turnover in Osteoporosis. Osteoporosis International, 6, S2-S17. http://dx.doi.org/10.1007/s001980070002

[16] Nicodemus, K.K. and Folsom, A.R. (2001) Type 1 and Type 2 Diabetes and Incident Hip Fractures in Postmenopausal Women. Diabetes Care, 24, 1192-1197. http://dx.doi.org/10.2337/diacare.24.7.1192 